\title{
Die Ausgestaltung der parlamentarischen Agendamacht - Plädoyer für eine holistische Analyse von Parlamenten
}

\author{
Michael Koß
}

The Determinants of Parliamentary Agenda Powers - A Holistic Framework for Analysing Parliamentary Change

Abstract: This article calls for a holistic perspective on parliamentary change through the analytical lens of parliamentary agenda powers. In addition to agenda control, the concept of agenda powers not only refers to the ability of parliamentary actors to directly influence parliamentary output through legislation (efficiency), but also to exert indirect influence through controlling the government (effectiveness) and through affecting public opinion (legitimacy). The concept of agenda powers comprises the formal institutional rules underlying the distribution of the parliamentary agenda, the factual application of these rules and the organisational powers enabling actors to make use of their time. The distribution of agenda powers can be explained by the sequencing of legitimacy, effectiveness, and efficiency reforms. Consequently, the concept of parliamentary agenda powers allows for a comparative long-term analysis of parliamentary change. Furthermore, the concept of parliamentary agenda powers enables us to operationalise normative scenarios of a de-parliamentarisation and the alleged transitions towards post- or neo-parliamentarian democracy.

Keywords: Parliaments, Agenda Control, Agenda Powers, Parliamentary Administrations, Sequencing

Schlagwörter: Parlamente, Agendakontrolle, Agendamacht, Parlamentsverwaltungen, Sequenzierung

\section{Einleitung'}

Die These vom Niedergang der Parlamente stellte das dominierende Paradigma der Parlamentarismusforschung im 20. Jahrhundert dar (Flinders u. Kelso 2011). Auch im beginnenden 21. Jahrhundert reißen die Klagen über eine Entmachtung der Volksvertretungen angesichts der zunehmenden internationalen Verflechtung und der daraus resultierenden Vorteile von Regierungen gegenüber Parlamenten nicht ab (vgl. exemplarisch Beisheim u. Brunnengräber 2008). In jüngster Zeit wurden die einschlägigen Befunde der Parlamentarismusforschung allerdings sowohl in normativer als auch empirischer Hinsicht zusehends differenziert.

Auf der normativen Ebene ist hier zum einen der Befund eines Übergangs in die postparlamentarische Demokratie zu nennen, der einen grundlegenden Wandel von Parlamenten impliziert (Andersen u. Burns 1996; Benz 1998). Aus dieser Per-

1 Für ihre Anmerkungen zu früheren Versionen dieses Aufsatzes danke ich Klaus H. Goetz und Simon Franzmann. Ebenso möchte ich mich bei den beiden anonymen Gutachtern bzw. Gutachterinnen und bei der Redaktion der PVS für ihre hilfreichen Hinweise bedanken. 
spektive kommen Parlamente in Mehrebenensystemen nicht umhin, Einbußen bei ihrer Gesetzgebungs- und Kontrollfunktion hinzunehmen. Um diese Einbußen zu kompensieren, wird eine Aufwertung der Öffentlichkeitsfunktion gefordert. Dem steht zum anderen die These eines Neoparlamentarismus gegenüber, die eine eher inkrementelle Anpassung von Parlamenten an die Herausforderungen durch Mehrebenenpolitik unterstellt, insbesondere durch eine stärkere horizontale und vertikale „Parlamentsverflechtung“ (Marschall 2005b, S. 334, s. a. 2005a, S. 284311).

Im Hinblick auf empirische Untersuchungen stellt das Konzept der parlamentarischen Agendakontrolle eine konzeptionelle Innovation in der Parlamentarismusforschung dar, die es ermöglicht, Parlamente systematisch zu analysieren, ohne ihre institutionelle Vielgestaltigkeit und die daraus resultierenden Ambivalenzen zu stark zu vereinfachen. Die grundlegende Annahme ist dabei, dass derjenige parlamentarische Akteur, der über die Zeitverteilung im parlamentarischen Gesetzgebungsprozess bestimmt, auch maßgeblichen Einfluss auf den parlamentarischen Output hat (Döring 1995). Die parlamentarische Tagesordnung kann auf verschiedene Art und Weise kontrolliert werden, sei es durch institutionelle Möglichkeiten ein Thema zu setzen (positive Agendakontrolle), Themen zu verhindern (negative Agendakontrolle) oder durch große Geschlossenheit im Abstimmungsverhalten und/oder ideologische Kohärenz (Cox 2006, S. 145; Tsebelis u. Rasch 2011, S. 2).

Insgesamt bietet sich in der Parlamentarismusforschung jedoch nach wie vor ein unbefriedigendes Bild: Konzeptionell wenig aufbereitete normative Befunde stehen stark spezialisierten empirischen Zugriffen gegenüber. Was fehlt, ist eine Heuristik, die beides zusammenbringt, die es also ermöglicht, durch systematische Analysen normative Befunde zu belegen oder zu verwerfen.

Zum Schließen dieser Lücke möchte ich einen Vorschlag unterbreiten. Im vorliegenden Beitrag werden drei Ergänzungen am Konzept der Agendakontrolle vorgeschlagen: Erstens plädiere ich dafür, nicht allein den direkten Einfluss des Parlaments auf den legislativen Output zu untersuchen, sondern auch den indirekten Einfluss, sei es durch die Kontrolle der Regierung oder durch Beeinflussung der Öffentlichkeit. Neben der gesetzgeberischen Effizienz sollten also zum einen die effektive Kontrolle und zum anderen legitimierende, auf Beeinflussung der Öffentlichkeit abzielende parlamentarische Diskurse als Möglichkeiten für parlamentarische Akteure aufgefasst werden, das Handeln der Exekutive zu beeinflussen. Zweitens sollte systematisch berücksichtigt werden, inwiefern formale Regeln der Zeitverteilung im Parlament auch faktisch angewendet werden und drittens, ob parlamentarischen Akteuren zur Nutzung ihrer Zeit die nötigen organisatorischen Ressourcen zur Verfügung stehen. Auf diese Weise ergibt sich das Konzept der parlamentarischen Agendamacht, welches es erlaubt, am Beispiel der Zeitverteilung im Plenum den Wandel von Parlamenten im Zeitverlauf und im internationalen Vergleich zu analysieren. Zudem vermag eine solch „holistische“ (McGann 2006, S. 458) Analyse von Parlamenten, die zunehmende ,Sprachlosigkeit ${ }^{*}$ zwischen empirischen und normativen Zugängen in der Parlamentarismusforschung zu überwinden. 
Im Folgenden wird zunächst das klassische Konzept der Agendakontrolle vorgestellt. Zudem erläutere ich die Probleme, die sich aus der mangelnden Berücksichtigung der faktischen Anwendung formaler Regeln und der dazu zur Verfügung stehenden organisatorischen Ressourcen ergeben. In Abschnitt 3 führe ich aus, dass parlamentarische Akteure das Handeln der Exekutive nicht allein direkt beeinflussen können (durch Mitwirkung an der Gesetzgebung), sondern auch indirekt, d. h. durch Kontrollaktivitäten oder Einwirken auf die öffentliche Meinung (die ja ihrerseits das Handeln der Regierung beeinflusst). Auf der Grundlage von Indikatoren für die jeweiligen Motive für eine Reform der Verteilung parlamentarischer Zeit werden in Abschnitt 4 Erwartungen dazu formuliert, wie sich ein Primat der Legitimität, Effektivität bzw. Effizienz auf die Ausgestaltung der Agendamacht auswirkt. Eine zentrale Rolle für die Ausgestaltung der Agendamacht kommt der Sequenzierung der jeweiligen Leitmotive zu, also der Frage, ob gleichzeitig unter dem Gesichtspunkt der Legitimität und der Effektivität oder Effizienz über die Verteilung parlamentarischer Zeit verhandelt wird oder ob Reformen zeitlich getrennt allein im Hinblick auf eines dieser Motive diskutiert werden (Abschnitt 5). Wird parlamentarische Zeit vornehmlich auf legitimierende Diskurse verwendet, so hat dies potenziell andere Folgen als ein Fokus auf die effektive Kontrolle der Regierung oder effiziente Gesetzgebung. Im ersteren Fall dürfte die parlamentarische Mehrheit bei der Zeitverteilung kaum privilegiert werden, im letzteren sehr wohl. Anstelle einer systematischen empirischen Überprüfung - die im Rahmen dieses Beitrags nicht geleistet werden kann - setze ich im sechsten Abschnitt das Konzept der Agendamacht in Bezug zu den beiden eingangs erwähnten normativen Thesen eines Post- bzw. Neoparlamentarismus. Im siebten Abschnitt werden die Ergebnisse zusammengefasst und die nächsten Schritte auf dem Weg zu einer holistischen Analyse von Parlamenten skizziert.

\section{Das Konzept der parlamentarischen Agendakontrolle}

Die Regeln der Agendakontrolle sind Teil der Parlamentsregeln, die als „formal rules and their authoritative interpretations that govern the conduct of politics in the parliamentary arena, including parliament's relations with other institutions" (Sieberer et al. 2011, S. 950) definiert werden können (s. a. Müller 2002, S. 280281). Im Mittelpunkt stehen dabei diejenigen Regeln, die über Entscheidungsmechanismen (Abstimmungsagenda) und den Zugang zum Plenum des Parlaments (Zeitplanagenda) bestimmen (Döring 2005, S. 111-115). Regeln der Abstimmungsagenda beziehen sich auf Politikinhalte (genauer: Gesetzesvorhaben), Regeln der Zeitplanagenda richten den Fokus auf die Verteilung parlamentarischer Zeit. Agendakontrolle ist entweder positiv, d. h., sie stellt sicher, dass ein Vorschlag bzw. Thema im Plenum behandelt bzw. darüber abgestimmt wird, oder negativ, d. h., sie verhindert, dass genau dies geschieht (Cox 2006, S. 149; Tsebelis u. Rasch 2011, S. 5-7). Mechanismen der Kontrolle der Abstimmungsagenda sind nicht nur das Recht, bestimmte Themen auf die parlamentarische Tagesordnung zu setzen oder sie von dieser zu streichen, sondern auch die Möglichkeit, Abstimmungen mit Vertrauensfragen zu verknüpfen oder - die subtilere Variante - Änderungsanträge zu Gesetzesvorschlägen auszuschließen. Die Zeitplanagenda kann 
beispielsweise durch die Möglichkeit, Debatten per Mehrheitsbeschluss zu beenden (die sogenannte „Guillotine“), kontrolliert werden.

Das Konzept der Agendakontrolle ist hier deshalb von besonderem Interesse, weil es zur Lösung von zwei Problemen beiträgt, die insbesondere die Analyse des Wandels von Parlamenten erschweren (McGann 2006, S. 444, 454-455). Zum einen das Problem der funktionalen Äquivalenz, denn aus der Perspektive der Agendakontrolle spielt es eine nachgeordnete Rolle, welche institutionelle Regel betrachtet wird. Wichtig ist allein ihr Zweck. Zum anderen trägt das Konzept der Agendakontrolle dem Problem des in parlamentarischen Systemen ja nur vermeintlichen Gegensatzes von Exekutive und Legislative Rechnung, wird hier doch nicht davon ausgegangen, dass alle Parlamentarier notwendigerweise identische Präferenzen haben. Innerparlamentarische Allianzen werden im Gegenteil als flexibel dargestellt, insbesondere die Interessen von parlamentarischer Mehrheit und Minderheit können in ihrem Verhältnis zueinander analysiert werden.

Untersuchungen zur parlamentarischen Agendakontrolle fokussieren in der Regel auf legislative Aktivitäten (z. B. Döring 1995; Cox u. McCubbins 2005, 2011; Tsebelis u. Rasch 2011). Allerdings kann Plenarzeit auch anderen Aktivitäten wie etwa der Kontrolle der Regierung (z. B. in Fragestunden) oder generellen Aussprachen über Themen von allgemeiner Bedeutung gewidmet sein. Diese sollten insbesondere in parlamentarischen Regierungssystemen von Bedeutung sein, in denen legislative Aktivitäten stärker als in präsidentiellen Systemen an die Regierung und die Ministerialbürokratie delegiert sind (Laver u. Shepsle 1996, S. 4, 57-58). Solange allerdings in Untersuchungen der parlamentarischen Tagesordnung die parlamentarische Zeit, die für andere als dezidiert legislative Aktivitäten vorgesehen ist, nicht berücksichtigt wird, ist das Konzept der Agendakontrolle nicht geeignet, holistische Analysen von Parlamenten anzuleiten. Die notwendige Erweiterung werde ich im folgenden Abschnitt vornehmen. Zuvor sollen jedoch zwei kleinere Ergänzungen angemahnt werden.

Wenn das Konzept der Agendakontrolle Analysen des parlamentarischen Wettbewerbs anleiten soll, so dürfen diese Analysen erstens nicht allein auf formale Regeln beschränkt bleiben. Neben informellen Regeln sollte vor allem der Frage nachgegangen werden, ob formale Regeln faktisch auch angewendet werden. Sheingate (2010) konnte am Beispiel des US-Kongresses zeigen, dass die Agendakontrolle einem faktischen Wandel unterliegt, der sich nicht in den formalen Regeln niederschlägt. Die wichtige Rolle, die der Frage nach der Anwendung von formalen Regeln zukommt, lässt sich am deutschen Beispiel verdeutlichen. Die weitreichende Mitsprache der Minderheit bei der Festlegung der parlamentarischen Agenda basierte vor allem auf informellen Absprachen. Ihre formalen Rechte hat die Minderheit im Bundestag bis 1983 hingegen kaum wahrgenommen. Als die Grünen und später die PDS von diesen Regeln verstärkt Gebrauch machten, wurden diese nicht etwa geändert, vielmehr passten sich die parlamentarischen Neulinge sukzessive dem Verhalten der etablierten Parteien an (Hoffmann Slagter u. Loewenberg 2009). Allein auf formale Regeln fokussierende Snapshot-Analysen können also schwerlich ermitteln, wie institutionelle Möglichkeiten im Hin- 
blick auf die Kontrolle der Agenda tatsächlich eingesetzt werden (s. a. Zubek 2011). ${ }^{2}$

Zweitens macht es eine Analyse der faktischen Anwendung formaler Regeln nötig, auch die organisatorischen Ressourcen zu berücksichtigen, die Akteuren zur Nutzung ihrer parlamentarischen Zeit Verfügung stehen. Organisatorische Aspekte der Entwicklung von Parlamenten wurden bislang eher stiefmütterlich behandelt. Mit Ausnahme der Literatur zur Institutionalisierung von Parlamenten (z. B. Polsby 1968; Hibbing 1988) - die allerdings organisationstheoretisch wenig fundiert und zudem von funktionalistischen Prämissen geleitet ist (Judge 2003; Huber 1996, S. 10-11) - verweist der Begriff legislative organisation traditionell nur auf die institutionellen Regeln innerhalb von Parlamenten (vgl. Strøm 1995). Auch ist der Zusammenhang zwischen verschiedenen Niveaus der Professionalisierung von Parlamenten (Borchert 2003; Z'Graggen 2009) und der Agendakontrolle bislang nicht untersucht worden. Dies ist jedoch nötig, da es gerade die organisatorischen Ressourcen sind, die es Akteuren ermöglichen, institutionelle Regeln für sich zu nutzen (Patzelt 2005, S. 283). Beispielsweise ist darauf hingewiesen worden, dass Europaausschüsse häufig nicht über die Ressourcen verfügen, die es ihnen ermöglichen, von ihren Rechten Gebrauch zu machen (vgl. Auel 2005, S. 306-307). In ähnlicher Weise fehlt es dem schweizerischen Parlament an organisatorischen Voraussetzungen dafür, Politikergebnisse zu beeinflussen (Schwarz et al. 2011, S. 135).

\section{Das Konzept der Agendamacht: Legitimität, Effektivität und Effizienz als Leitmotive der Verteilung parlamentarischer Zeit}

Wenn auf der Grundlage des Konzepts der parlamentarischen Agendakontrolle die Entwicklung von Parlamenten holistisch analysiert werden soll, so gilt es, den exklusiven Fokus der klassischen Agendakontrolle über den direkten Einfluss des Parlaments auf das Handeln der Regierung hinaus zu erweitern. Döring (2005, S. 110) unterscheidet neben der Zeitplan- und der Abstimmungsagenda auch die außerparlamentarische Themenagenda, d. h. die Beeinflussung des öffentlichen Diskurses durch das Parlament, diskutiert diese aber nicht näher. Es liegen eine Reihe von Untersuchungen zur Fähigkeit politischer Akteure, die öffentliche Agenda zu bestimmen, vor. Bemerkenswerterweise wurden diese Untersuchungen zum Agenda Setting bislang nicht systematisch mit Analysen der (wie erwähnt vor allem auf legislative Tätigkeiten fokussierenden) Agendakontrolle in Beziehung gesetzt (Majone 2006). Just dies soll im Folgenden geschehen. Angesichts der weitreichenden Änderungen, die sich daraus (und aus der Berücksichtigung der Anwendung formaler Regeln sowie der Akteuren zur Nutzung ihrer Zeit zur Ver-

2 Diesem Desiderat tragen jüngst auch Tsebelis und Rasch (2011, S. 11) Rechnung, die in ihrer Analyse der Agendakontrolle nicht allein formale Regeln berücksichtigen, sondern auch parteiliche (die Stabilität von Regierungen) und positionelle Faktoren (ideologische Präferenzen). Ihnen zufolge sind positionelle und parteiliche Faktoren umso wichtiger, je geringer das Ausmaß der formal-institutionellen Agendakontrolle ist. Wenn also Koalitionspartner über keine institutionellen Vorteile verfügen, kommt es maßgeblich auf deren ideologische Distanzen und die Abstimmungsdisziplin an. Dies ist eigentlich nichts anderes als ein Plädoyer für eine stärkere Berücksichtigung der Frage nach der faktischen Anwendung formaler Regeln. 
fügung stehenden organisatorischen Ressourcen) ergeben, erscheint es gerechtfertigt, anstelle von Agendakontrolle von parlamentarischer Agendamacht zu sprechen. Erst die integrierte - und in diesem Sinne holistische - Analyse der parlamentarischen Agendamacht kann dazu beitragen, am Beispiel der Zeitverteilung im Plenum den Wandel von Parlamenten systematisch zu analysieren.

Wie erwähnt fokussiert das klassische Konzept der Agendakontrolle exklusiv auf die Frage, wie parlamentarische Akteure das Handeln der Exekutive im Bereich der Gesetzgebung direkt beeinflussen (Schaubild 1a). Wird parlamentarische Zeit unter dem Primat der Gesetzgebung (beispielsweise also für die verschiedenen Lesungen von Gesetzesentwürfen im Plenum des Parlaments) vergeben, stehen Fragen der parlamentarischen Effizienz im Vordergrund: Wie viel Einfluss haben parlamentarische Akteure auf die in der Regel von der Exekutive eingebrachten Gesetzesentwürfe? Je mehr das Plenum des Parlaments über die Vergabe parlamentarischer Zeit entscheidet, desto größer ist nach dieser Lesart der parlamentarische Einfluss (Döring 1995). ${ }^{3}$ Am geringsten ist der Einfluss dann, wenn allein die (Regierungs-)Mehrheit über die Gesetzgebungsagenda entscheiden kann.

Schaubild 1: Agendakontrolle und Agendamacht

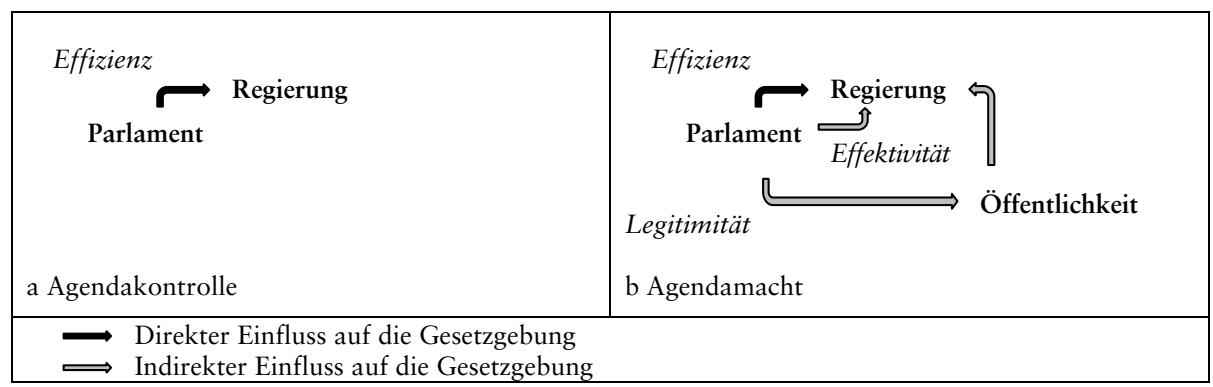

Allerdings gibt es auch indirekte Wege für parlamentarische Akteure, das Handeln der Regierung zu beeinflussen (Schaubild 1b). Erstens können parlamentarische Akteure dadurch Einfluss auf die Regierung ausüben, dass sie diese kontrollieren, parlamentarische Zeit also investigativen Aktivitäten widmen (z. B. Fragestunden mit anschließenden Abstimmungen). Bedingung dafür ist allerdings analog zur parlamentarischen Zeit für Gesetzgebung, dass nicht allein die Mehrheit über die Agenda entscheidet. Auf diese Weise dürften parlamentarische Kontrollaktivitäten durch antizipatorische Effekte indirekten Einfluss auf die Gesetzgebung ausüben: Eine Regierung, die weiß, dass parlamentarische Akteure ihr Handeln reglementieren können (sei es ex ante oder ex post), wird dies in ihrem gesetzgeberischen Handeln erwartungsgemäß berücksichtigen (Sieberer 2011, S 736). In diesem Fall steht bei der Verteilung parlamentarischer Zeit also das Ziel im Mittelpunkt, effektiven (kontrollierenden) Einfluss auf Regierungen zu nehmen.

3 Damit ist nicht gesagt, dass dieser Einfluss im Plenum ausgeübt wird. Wenn das Plenum des Parlaments umfassenden Einfluss auf die Gesetzgebungsagenda hat, wird dieser Einfluss in der Regel an Ausschüsse delegiert. 
Zweitens können parlamentarische Akteure das gesetzgeberische Handeln von Regierungen indirekt beeinflussen, indem sie auf die außerparlamentarische Themenagenda (also die öffentliche Meinung) einwirken, die - vermittelt durch die Medien oder Umfragen - wiederum exekutives Handeln beeinflussen kann (s. a. McGann 2006, S. 457). Soll die außerparlamentarische Themenagenda systematisch berücksichtigt werden, so ergibt sich eine weitere Maßgabe, unter der parlamentarische Zeit vergeben werden kann: Neben die exklusiv auf die Beziehungen zwischen Parlament und Regierung fokussierende Effizienz und Effektivität tritt das Ziel, die Legitimität des Parlaments zu erhöhen. Legitimität ermöglicht es parlamentarischen Akteuren, exekutives Handeln indirekt - auf dem Weg über die Öffentlichkeit - zu beeinflussen (Schaubild 1b). Wird parlamentarische Zeit legitimitätsstiftenden Aktivitäten - ,großen', idealerweise ergebnisoffenen Aussprachen über zentrale politische Themen - gewidmet, dann erhöht dies die Fähigkeit parlamentarischer Akteure, die öffentliche Meinung zu beeinflussen (und damit indirekt auch das Handeln der Regierung). Insbesondere in parlamentarischen Systemen bietet sich diese Strategie durchaus an, verfügen doch allein die Parlamente qua Volkswahl über eine direkte Legitimationsbasis, die sich als politisches Pfund erweisen kann, wenn ihrer Pflege parlamentarische Zeit gewidmet ist. Vor diesem Hintergrund erscheint es wenig angemessen, dass auch in jüngeren und elaborierteren Analysen der Macht von Parlamenten die Rolle der parlamentarischen Legitimität nicht näher erörtert wird (z. B. Sieberer 2011, S. 749).

Die Agendamacht von Parlamenten wird also unter drei Gesichtspunkten vergeben, die den klassischen Parlamentsfunktionen Gesetzgebung, Kontrolle und Repräsentation (Kreppel 2011, S. 125) zugeordnet werden können: der gesetzgeberischen Effizienz von parlamentarischen Akteuren, ihrer Fähigkeit zur effektiven Kontrolle der Regierung und ihren Möglichkeiten, aus ihrer Stellung als direkt legitimierte Repräsentanten der Wähler politisches Kapital zu schlagen. Damit parlamentarische Akteure Agendamacht ausüben können, bedarf es nicht allein formeller Regeln. Um Macht zu entfalten, müssen diese Regeln darüber hinaus auch faktisch angewendet werden. Ferner müssen parlamentarischen Akteuren die zu diesem Zweck ggf. nötigen organisatorischen Ressourcen zur Verfügung stehen. All dies gilt es bei der Analyse der parlamentarischen Agendamacht zu berücksichtigen.

Im Folgenden sollen die drei Leitmotive der Ausgestaltung der parlamentarischen Agendamacht operationalisiert werden (Schaubild 2). Es werden Indikatoren entwickelt, mit deren Hilfe sich die einzelnen Zwecke, denen parlamentarische Zeit gewidmet werden kann, unterscheiden lassen. Auf der Grundlage dieser Operationalisierung kann im nächsten Abschnitt danach gefragt werden, welchen Einfluss welches Motiv auf die Verteilung parlamentarischer Zeit, die Anwendung der jeweiligen Regeln und die organisatorischen Ressourcen, die parlamentarischen Akteure dazu zur Verfügung stehen, hat.

Die wichtigsten Indikatoren der drei Leitmotive bei der Verteilung parlamentarischer Agendamacht sind zunächst die jeweils handelnden Akteure und ihre Adressaten. Darüber hinaus spielt es eine Rolle, in welcher Arena über die Ausgestaltung der Agendamacht verhandelt wird und welches inhaltliche Ziel die Akteure an eine Reform der Zeitverteilung im Parlament knüpfen. Eng damit verknüpft 
ist, wofür parlamentarische Zeit und Ressourcen verwendet werden sollen und inwiefern Entscheidungen über die parlamentarische Agendamacht mit anderen parlamentarischen Reformen verbunden sind. Schließlich unterscheiden sich Maßgaben der Legitimität, Effektivität und Effizienz hinsichtlich der ihnen zugrundeliegenden Interaktionsorientierungen. Je nachdem, ob Akteure solidarischen oder individuellen Handlungsprinzipien folgen und ähnliche oder verschiedene Ziele verfolgen, lässt sich hier zwischen Kooperation, Verhandlung, Wettbewerb oder Konflikt unterscheiden (Bartolini 1999; 2000). ${ }^{4}$

\section{Schaubild 2: Leitmotive der Verteilung parlamentarischer Agendamacht und ihre Operationalisierung}

\begin{tabular}{|c|c|c|c|}
\hline & Legitimität & Effektivität & Effizienz \\
\hline (kollektive) Akteure & alle Parlamentarier & $\begin{array}{l}\text { parlamentarische } \\
\text { Minderheit }\end{array}$ & $\begin{array}{l}\text { Fraktionen / } \\
\text { Parteien }\end{array}$ \\
\hline Adressat & Souverän & $\begin{array}{l}\text { (Regierungs-) Mehrheit } \\
\text { \& Souverän }\end{array}$ & andere Fraktionen \\
\hline Arena & $\begin{array}{l}\text { extern: } \\
\text { Parlament - } \\
\text { Souverän }\end{array}$ & $\begin{array}{l}\text { quasi-extern: } \\
\text { Parlament - } \\
\text { Regierung }\end{array}$ & $\begin{array}{l}\text { intern: } \\
\text { Parlament }\end{array}$ \\
\hline Inhalt & Kommunikation & Kontrolle & Gesetzgebung \\
\hline $\begin{array}{l}\text { Zeit und Ressourcen } \\
\text { werden verwendet für }\end{array}$ & Diskurs & Untersuchung & Anträge \\
\hline $\begin{array}{l}\text { oft verbunden mit } \\
\text { Entscheidungen über }\end{array}$ & $\begin{array}{l}\text { Außenbeziehungen } \\
\text { des gesamten } \\
\text { Parlaments }\end{array}$ & $\begin{array}{l}\text { parlamentarische } \\
\text { Fragerechte, Vertrau- } \\
\text { ensabstimmungen }\end{array}$ & $\begin{array}{l}\text { Rechte von } \\
\text { Ausschüssen }\end{array}$ \\
\hline $\begin{array}{l}\text { Interaktionsorien- } \\
\text { tierung }\end{array}$ & Kooperation & Konflikt & $\begin{array}{l}\text { Verhandlung / } \\
\text { Wettbewerb }\end{array}$ \\
\hline
\end{tabular}

Reformen der parlamentarischen Agendamacht können erstens dem Ziel dienen, die parlamentarische Legitimität zu stärken und so indirekt das Handeln der Regierung zu beeinflussen. Der Begriff der Legitimität bezieht sich auf jedwede Argumente zur Begründung politischer Autorität, die zur Durchsetzung von verbindlichen Entscheidungen benötigt wird (Scharpf 2000, S. 102; Hardin 2007). Bei Legitimitätsreformen stehen also die einzelnen Parlamentarier als gleichberechtigte Akteure im Mittelpunkt, verfügen diese doch alle über dieselbe demo-

4 Von Kooperation und Verhandlung kann dann gesprochen werden, wenn Akteure sich solidarisch verhalten, d. h. ihr Handeln koordinieren. Im Fall der Kooperation streben sie zudem nach ähnlichen inhaltlichen Zielen. Bei der Verfolgung ihrer Interessen verzichten sie nicht nur auf Zwang ihren Konkurrenten gegenüber, sondern teilen sogar wichtige Informationen, da sie davon ausgehen, dass sie ihr Ziel nur gemeinsam erreichen können. Es herrscht also keine the winner takes it all-Mentalität vor. Während kooperierende Akteure Informationsressourcen teilen, arbeiten verhandelnde Akteure mit Versprechen und Drohungen. Konfliktorientierte Akteure folgen ebenso wie konkurrierende individuellen Handlungsprinzipien. Es kommt also zu keinerlei wechselseitiger Koordination, im Falle von Konflikt aufgrund verschiedener Ziele der Akteure, im Falle von Konkurrenz trotz ähnlicher Ziele (vgl. zusammenfassend Bartolini 1999, S. 444). 
kratische Legitimation. Der Adressat von Legitimitätsreformen ist der Souverän, ${ }^{5}$ denn Legitimität entspricht einer symbolischen Macht, die erst durch Kommunikation nach außen hergestellt wird. Diese Kommunikation ist ihrerseits Grundlage der Fähigkeit des Parlaments zu responsivem Handeln und Repräsentation (Patzelt 2001, S. 56-68). Soll die parlamentarische Legitimität erhöht werden, werden Zeit und Ressourcen des Parlaments folglich darauf verwendet, einen Diskurs zu führen, der diese mit der Wählerschaft verbindet. Solche Diskurse dienen dazu, politische Ideen und Werte zu kommunizieren (Schmidt u. Radaelli 2004, S. 184). Sie bilden den Rahmen der nationalen politischen Diskussion, indem sie politische Ereignisse erklären, politische Aktionen legitimieren, politische Identitäten entwickeln und politische Geschichte reinterpretieren (March u. Olsen 1995, S. 45, 66). Diskurse sind idealerweise deliberativer Natur, denn Legitimität kann am besten durch Überzeugung gewährleistet werden (Scharpf 2000, S. 104, 117-120). Jenseits der Auseinandersetzung über die Zeitverteilung im Plenum dürften Legitimitätsreformen auch die Belange des gesamten Parlaments (und weniger seiner partikularen Bestandteile wie etwa den Fraktionen) berühren, vor allem dessen Außenbeziehungen (vgl. Marschall 1999b). Solche Reformen werden in der Literatur zu Parlamentsreformen als „Öffnung“ (Blumenthal 2009, S. 18) oder „Verkopplung von Parlament und Öffentlichkeit“ (Marschall 1999a, S. 75) bezeichnet. Streben parlamentarische Akteure nach Erhöhung ihrer Legitimität, also der Anerkennung durch die Wählerschaft, so ist dies schwerlich mit kompetitiven Orientierungen vereinbar, denn Anerkennung beruht auf Kooperationsbereitschaft. Honneth (2011, S. 38) spricht von einem dem Streben nach Anerkennung inhärenten „Zwang zur Reziprozität“. Aus diesem Grund dürften Parlamentarier in Legitimitätsreformen kooperativ handeln.

Anders als bei Legitimitäts- sind bei Effektivitätsreformen nicht die einzelnen Parlamentarier, sondern die jeweilige Mehrheit und die Minderheit die maßgeblichen Akteure. An parlamentarischer Zeit zur Erhöhung der Effektivität sind vor allem Minderheitsakteure interessiert, die danach streben, der Mehrheit Fehler nachzuweisen. Dementsprechend wird parlamentarische Zeit stärker für die innerparlamentarische Auseinandersetzung verwendet als bei Reformen, bei denen die Erhöhung der Legitimität des Parlaments im Vordergrund steht. Dennoch implizieren Effektivitätsreformen einen quasi-externen Charakter der Auseinandersetzung, wird die Frage der Kontrolle exekutiven Handelns doch meist als Gegensatz zwischen „dem Parlament“ und „der Regierung“ zugespitzt, und dies ungeachtet der Tatsache, dass letztere aus ersterem hervorgeht. Deshalb ist hier ähnlich wie bei Legitimitätsreformen zu erwarten, dass Parlamentarier nach außen gerichtet argumentieren und die Auseinandersetzung nicht allein als innerparlamentarisch betrachten. Parlamentarische Zeit wird in diesem Fall für Untersuchungen, d. h. Aussprachen über Aktivitäten der Regierung, verwendet. Daraus folgt, dass Effektivitätsreformen mit Entscheidungen über parlamentarische Ex-

5 Der Begriff „Souverän“ wurde hier gewählt, um dem Wandel des Legitimitätskonflikts im Zeitverlauf gerecht zu werden: In konstitutionellen Systemen waren es vornehmlich Monarchen, um deren Anerkennung es Parlamenten ging. Unter den Bedingungen der Demokratie ist dies die Wählerschaft. 
post- oder Ex-ante-Kontrolle, d. h. Fragerechte (Schnapp u. Harfst 2005; Russo u. Wiberg 2010) oder Wahlbefugnisse (Sieberer 2010, S. 139-63), verbunden sind. Da die Ziele von regierungsnaher Mehrheit und regierungsferner Minderheit in Effektivitätsreformen einander grundsätzlich ausschließen, dürfte ihre Interaktionsorientierung die des Konflikts sein. Nichtsdestotrotz kann dieser strukturelle Bias, der in Richtung eines Dissenses zwischen Mehrheits- und Minderheitsakteuren wirkt, auf zweierlei Weise überkommen werden: zum einen, wenn im Fall von Minderheitsregierungen (Strøm 1990) die klassischen Mehrheitsverhältnisse umgekehrt sind, die regierende Minderheit also auf die Kooperation der oppositionellen Mehrheit angewiesen ist; zum anderen dadurch, dass ein Minimum an Kontrolle der Regierung generell demokratietheoretisch geboten ist. Wenn es gilt, dieses Minimum festzulegen, dürften in der Regel aber Konflikte zwischen Mehrheit und Minderheit entstehen.

Wir haben bereits gesehen, dass Fragen der parlamentarischen Effizienz, also der Gesetzgebung, vornehmlich die genuin parlamentarische Arena berühren. Hier steht der Output des Parlaments direkt zur Debatte. Allerdings verläuft die Auseinandersetzung über Gesetzgebungsanträge anders als im Fall von Effektivitätsreformen nicht allein zwischen Mehrheit und Minderheit. Zumindest im Fall von Koalitionsund Minderheitsregierungen sind inhaltliche Differenzen auch innerhalb der ein Gesetz tragenden Mehrheit denkbar. Deshalb dürften die Fraktionen die maßgeblichen Akteure bei Entscheidungen über die Verteilung parlamentarischer Zeit für Gesetzgebungsaktivitäten sein. Die Fraktionen als Vereinigungen von parlamentarischen Akteuren mit ähnlichen Policy-Präferenzen sind in Effizienzreformen Akteure und Adressaten. Die Öffentlichkeit hingegen spielt bei diesen direkten Entscheidungen über Politikinhalte eine nachgeordnete Rolle. Entscheidungen, die eng mit Effizienzreformen verbunden sein können, firmieren in der Literatur zu Parlamentsreformen unter dem Label der „Modernisierung“ (Flinders 2007, S. 179), d. h. der Versuche, den legislativen Output von Parlamenten zu verbessern. Da Detailfragen der Gesetzgebung in der Regel vom Plenum des Parlaments delegiert werden, erwarte ich, dass Effizienzreformen vor allem mit Entscheidungen über die Einsetzung bzw. die Befugnisse von parlamentarischen Ausschüssen (Mattson u. Strøm 1995) verbunden sind. Fragen der Gesetzgebung eröffnen mannigfaltige Möglichkeiten zu Koppelgeschäften, weshalb Effizienzreformen flexiblere Interaktionsorientierungen zugrunde liegen sollten, die zwischen Verhandlung (wenn Akteure verschiedene Ziele verfolgen, aber solidarisch handeln, z. B. in Koalitionen) und Wettbewerb (wenn Akteure ähnliche Ziele verfolgen, aber nicht solidarisch handeln, z. B. im Fall von Minderheitsregierungen) changieren.

\section{Auswirkungen von Legitimitäts-, Effektivitäts- und Effizienzreformen auf die Ausgestaltung der Agendamacht}

Im Mittelpunkt der Analyse der Auswirkungen verschiedener Reformarten stehen folgende Aspekte: erstens die Proportionalität der Verteilung parlamentarischer Zeit, also die Frage, ob und inwiefern Mehrheits- oder Minderheitsakteure privilegiert werden; zweitens der Aufbau und der Umfang der organisatorischen Res- 
sourcen, die parlamentarischen Akteuren zur Nutzung ihrer jeweiligen Rechte zur Verfügung stehen und drittens den Zusammenhang zwischen der parlamentarischen Agendamacht und ihrem institutionellen Kontext.

Um organisatorische Ressourcen von parlamentarischen Akteuren zu unterscheiden, greife ich auf Brunssons (1989, S. 15-21) Gegenüberstellung von "Handlungsorganisationen“ (action organisations) und „politischen“ Organisationen zurück. Brunsson zufolge stehen Organisationen zwei idealtypische Möglichkeiten offen, ihren Fortbestand zu sichern: Sie können entweder den Erwartungen ihrer Umwelt genügen oder konkrete Leistungen erbringen (s. a. Meyer u. Rowan 1977). Handlungsorganisationen sind vor allem an letzterem interessiert. Sie verfügen über eine starke organisatorische Ideologie, die eine Spezialisierung und koordiniertes Handeln auch in unvorhergesehenen Situationen erlaubt. Zustimmung gilt in politischen Organisationen als Rekrutierungs- und Organisationsprinzip, um sicherzustellen, dass alle Mitglieder ähnliche Ziele verfolgen. Diese strikte Ideologieorientierung stellt sicher, dass Handlungsorganisationen lösungsbezogen agieren - konkurrierende Ansätze werden schlicht ausgeblendet, sodass zwischen Ideologie und Handeln kein Widerspruch besteht. Der Gegentypus zu Handlungs- sind politische Organisationen, die ideologische Inkonsistenzen zulassen und ausgleichen - allerdings auf Kosten ihrer Handlungsfähigkeit. Politische Organisationen sind deshalb durch ein geringes Maß an Integration gekennzeichnet. Konflikte sind wichtig für die Mitglieder politischer Organisationen, die sich oftmals als Anhänger ideologisch verschiedener Suborganisationen ansehen. Politische Organisationen ,erzeugen` vor allem talk im Gegensatz zur action der Handlungsorganisationen (Brunsson 1989, S. 26). ${ }^{6}$

Das von Legitimitäts-, Effektivitäts- und Effizienzreformen implizierte Verhältnis der parlamentarischen Agendamacht zum institutionellen Kontext des jeweiligen politischen Systems ist hier deshalb von Bedeutung, weil es Rückschlüsse auf die Anwendung der Regeln der Agendamacht zulässt. Um zu ermitteln, inwiefern vorhandene Rechte (insbesondere solche von Minderheiten) auch tatsächlich angewendet werden, soll hier der Frage nachgegangen werden, ob die parlamentarische Arena jeweils an andere den politischen Wettbewerb beeinflussende (Verfassungs-)Institutionen wie zweite Kammern, Verfassungsgerichte, Referenden etc. eng oder lose gekoppelt ist. Während Entscheidungen in eng gekoppelten Institutionen wechselseitig Entscheidungszwänge verursachen, ist dies bei loser Kopplung nicht der Fall (Weick 1985, S. 162-165). Bei loser Kopplung dienen solche Entscheidungen vielmehr als Argumente und Informationen, auf deren Grundlage Entscheidungen getroffen werden können (s. a. Benz 1998, S. 216-218). Daraus lässt sich schließen, dass vor allem eine lose Kopplung die faktische Anwendung vorhandener Rechte, insbesondere von parlamentarischen Minderheitsakteuren, befördert. Bei loser Kopplung müssen anderswo ausgehandelte Lösungsvorschlä-

6 Interessanterweise sieht Brunsson Parlamente als typische Beispiele für „politische“ Organisationen an. Dies spielt hier aber eine nachgeordnete Rolle, da Brunsson sich dabei ähnlich wie der Mainstream der Parlamentarismusforschung auf die Ebene der (nach ideologischen Kriterien organisierten) Abgeordneten bezieht, nicht aber der diesen zur Verfügung stehenden organisatorischen Ressourcen, um die es hier geht. 
ge nicht bedingungslos akzeptiert werden, sondern können im Parlament neu verhandelt werden. ${ }^{7}$ Das Konzept der Kopplung hat zudem den Vorteil, dass sich mit seiner Hilfe das Verhältnis von parlamentarischer Agendamacht und ihrem institutionellen Kontext differenzierter analysieren lässt als durch einen Fokus auf die dichotome Unterscheidung zwischen Endogenität und Exogenität, der die Debatte über die Entwicklung der Agendakontrolle dominiert. ${ }^{8}$

Wie im Folgenden zu zeigen sein wird, besteht vor allem zwischen den Konsequenzen von Legitimitäts- und Effizienzreformen ein Gegensatz hinsichtlich der von ihnen implizierten Verteilung von Zeit und Ressourcen sowie der Anwendung von Regeln. Effektivitätsreformen nehmen hingegen eine Mittelposition ein (Schaubild 3).

Wie im vorangegangenen Abschnitt dargelegt, dienen Legitimitätsreformen vor allem dem Diskurs des Parlaments mit der Öffentlichkeit, durch den letztere überzeugt werden und ihrerseits Einfluss auf die Regierung nehmen soll. Dies impliziert eine proportionale Verteilung von parlamentarischer Zeit, denn die Möglichkeit, die Öffentlichkeit zu überzeugen, ist notwendigerweise an eine sachliche und faire Debatte im Parlament gebunden. Wird aber die Mehrheit bei der Vergabe parlamentarischer Zeit privilegiert, ist schwerlich gewährleistet, dass die Öffentlichkeit sich durch eine von ihr einseitig dominierte Debatte überzeugen lässt. Anders formuliert müssen Legitimitätsreformen also den deliberativen ArguingModus des parlamentarischen Diskurses stärken und sicherstellen, dass dieser nicht in strategisches bargaining oder rein normengeleitetes Verhalten kippt (vgl. Risse 2000). Der grundlegende Unterschied zwischen diesen Formen kommunikativen Handelns ist, dass Akteure sich beim arguing ohne vollkommen fixe Präferenzen begegnen (also wechselseitig überzeugt werden können), während dies weder bei der strategischen Zielorientierung des bargaining noch bei rein normengeleitetem (und damit ebenfalls unflexiblem) Verhalten der Fall ist. Wech-

7 Den Vorteil loser Kopplung verdeutlicht das Beispiel des deutschen Bundestages: Ein Grund, warum hier auf majoritäre Regeln der Agendakontrolle bzw. deren Anwendung verzichtet wurde, ist die lose Kopplung des Bundestages an den Bundesrat. Insbesondere in Zeiten divergierender Mehrheiten in beiden Häusern ist es eben nicht so, dass Entscheidungen in einer der Kammern diejenigen in der anderen determinieren. Deshalb müssen bei ausbleibender Zustimmung des Bundesrates Beschlüsse des Bundestages erneut verhandelt werden (zumindest im Fall von zustimmungspflichtigen Gesetzgebungsmaterien). Prozedurale Vorteile der Bundestagsmehrheit sind in diesem Fall von nachrangiger Bedeutung (Sieberer 2006, S. 63-64). Die lose Kopplung beider Kammern befördert also konsensuelle und informelle Absprachen über die parlamentarische Agenda, mithin also die faktische Anwendung kodifizierter Rechte parlamentarischer Akteure.

8 Die Frage nach dem Einfluss externer (Verfassungs-)Regeln auf die parlamentarische Agendakontrolle ist in der Literatur umstritten. Sieberer et al. (2011) konnten Hinweise für einen Zusammenhang zwischen dem Wandel von Parlamentsregeln und der Europäisierung, den Konjunkturen der demokratischen Reformbewegung und der steigenden Komplexität politischer Themen feststellen. Dies kann ihnen zufolge als Beleg dafür angesehen werden, dass Parlamentsregeln nicht exogen zum politischen Wettbewerb sind und mithin nicht als stabil angesehen werden können (s. a. Cox 2000). Im Gegensatz dazu geht etwa Taylor (2006) davon aus, dass parlamentarischen Minderheiten in großen, unikameralen oder schwachen Zweikammerparlamenten nur eingeschränkte prozedurale Rechte zugestanden werden. Noch stärker gegen die Annahme einer Endogenität parlamentarischer Regeln zum politischen Wettbewerb argumentiert McCubbins (2005), dem zufolge die parlamentarischen Regeln der Gesetzgebung diejenigen der Verfassung lediglich spiegeln. Dazu passt der Befund von Powell (2000), der einen Zusammenhang zwischen der Proportionalität von Wahlsystemen und parlamentarischen Gesetzgebungsprozeduren postuliert. 
selseitig überzeugen lassen sich allerdings nur Akteure, die nicht den Eindruck haben, übervorteilt zu werden. Deshalb dürfte eine proportionale Verteilung parlamentarischer Zeit für legitimierende Diskurse unabdingbar sein. Aus Fairnessgründen wäre es lediglich möglich, Minderheiten maßvoll zu privilegieren.

Da wechselseitiges Überzeugen zudem an möglichst neutrale Information gebunden ist, erfordern Legitimitätsreformen den Aufbau einer Handlungsorganisation im Sinne Brunssons, die auf der Ebene des gesamten Parlaments (und nicht der partikularen Fraktionen) angesiedelt sein sollte. Ziel dieser Handlungsorganisation, die in ihrem Aufbau der sektoral strukturierten Ministerialverwaltung (Schnapp 2004) ähneln sollte, ist es dann, den parlamentarischen Akteuren diejenigen Informationen zur Verfügung zu stellen, die sie zur Überzeugung der Öffentlichkeit benötigen. Politische Organisationsressourcen sind dazu zumindest auf der Ebene des Parlaments nicht nötig. Parteiliche Informationen können stattdessen ggf. durch die Parteiorganisationen - die parties in central office (Katz u. Mair 1993) - bezogen werden. Hinsichtlich des Verhältnisses der parlamentarischen Arena zu ihrem institutionellen Kontext sind erfolgreiche Legitimitätsreformen an eine lose Kopplung gebunden, denn nur diese versetzt parlamentarische Akteure in die Lage, die nationale Agenda zu bestimmen. Eine zu starke interinstitutionelle Verschränkung dürfte diese Möglichkeit beschneiden, denn hier können andere, flexiblere Akteure wie Regierungen Entscheidungszwänge geltend machen und deliberative Diskurse gleichsam unterbinden. Dieses Problem tritt insbesondere in Mehrebenensystemen wie der EU auf, die durch eine enge Kopplung der Ebenen gekennzeichnet sind (Scharpf 2000, S. 113-115).

Schaubild 3: Erwarteter Zusammenhang zwischen parlamentarischer Agendamacht und den Leitmotiven der Legitimität, Effektivität und Effizienz

\begin{tabular}{|c|c|c|}
\hline Aspekte der Agendamacht & & Primat bei Reformen der Agendamacht \\
\hline $\begin{array}{l}\text { temporal } \\
\text { (a) Zeitverteilung } \\
\text { im Plenum } \\
\text { organisatorisch } \\
\text { (b) administrative } \\
\text { Ressourcen } \\
\text { kontextuell } \\
\text { (c) Kopplung mit } \\
\text { institutionellem } \\
\text { Kontext }\end{array}$ & 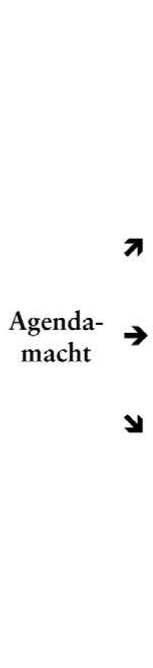 & $\begin{array}{l}\text { Legitimität } \\
\text { (a) je unabhängiger von anderen Reformzielen, desto } \\
\text { proportionaler } \\
\text { (b) Handlungsorganisation auf der Ebene des gesamten } \\
\text { Parlaments } \\
\text { (c) lose Kopplung } \\
\text { Effektivität } \\
\text { (a) je unabhängiger vom Ziel, die parlamentarische } \\
\text { Legitimität zu erhöhen, desto majoritärer; je } \\
\text { unabhängiger vom Ziel der Effizienzsteigerung, desto } \\
\text { proportionaler } \\
\text { (b) „Politische“ Organisationen auf der Ebene der } \\
\text { parlamentarischen Gruppen } \\
\text { (c) lose Kopplung } \\
\text { Effizienz } \\
\text { (a) je unabhängiger vom Ziel, die parlamentarische } \\
\text { Legitimität zu erhöhen, desto majoritärer } \\
\text { (b) Handlungsorganisationen auf der Ebene des } \\
\text { parlamentarischen Gruppen } \\
\text { (c) enge Kopplung }\end{array}$ \\
\hline
\end{tabular}


Im Gegensatz zu Legitimitätsreformen bieten die Ziele effektiver Kontrolle und effizienter Gesetzgebung sehr wohl Anlass dazu, parlamentarische Mehrheitsakteure zu privilegieren. Hinsichtlich der Verteilung parlamentarischer Zeit besteht bei Effektivitätsreformen zwar kein Anlass, von der Proportionalität abzuweichen, denn diese gewährleistet ja bereits, dass die Mehrheit der Regierung nicht überstimmt wird, Kontrolle also begrenzt bleibt. Anders sieht es bei der Ressourcenverteilung im Zuge von Effektivitätsreformen aus, die sich grundsätzlich von derjenigen unterscheidet, die ein Primat der Legitimität impliziert. Die Informationen, die politische Akteure zur Ausübung von Kontrolle benötigen, müssen überwiegend parteiisch sein, denn die Interessen von Mehrheit und Minderheit sind hier ideologisch determiniert. Anders lässt sich kaum erklären, warum beispielsweise der Deutsche Bundestag erst 2000, nach jahrzehntelanger Diskussion, ein Untersuchungsausschussgesetz verabschiedete. Zuvor waren die jeweiligen Regierungsund Oppositionsfraktionen schlicht zu zerstritten (Demuth 2009). Eine Erhöhung der parlamentarischen Effektivität gewährleisten am besten politische Organisationsressourcen, die auf der Ebene der Fraktionen angesiedelt sind. Ein politischer Organisationsaufbau sichert die Loyalität als die wichtigste Voraussetzung für eine effektive Kontrolle der anderen parlamentarischen Akteure unabhängig von der Frage, ob man deren Handeln nicht grundsätzlich billigt. Ebenso wie die parlamentarische Legitimität kann die Effektivität der Volksvertretung unter der Bedingung einer engen Kopplung der parlamentarischen Arena an ihren institutionellen Kontext begrenzt werden, wenn Mehrheitsakteure auf Handlungszwänge verweisen, die sich in anderen Arenen ergeben und sich so einer Ex-post-Kontrolle entziehen. Eine lose Kopplung befördert also auch die parlamentarische Effektivität.

Effizienzreformen hingegen ermöglichen es, parlamentarische Zeit ungleich zu verteilen. Mit dem Argument, die Fülle an zu verabschiedenden Gesetzgebungsvorhaben übersteige die vorhandene parlamentarische Zeit lässt sich eine Beschränkung der Zeit, die der Minderheit zur Verfügung steht, rechtfertigen. Cox (1987) zufolge ist genau dies der Grund, warum im britischen Unterhaus die Regierungsmehrheit sukzessive einen überproportionalen Anteil der parlamentarischen Zeit für sich (d. h. ihre Gesetzgebungsvorhaben) beanspruchen konnte. Hinsichtlich der organisatorischen Ressourcen des Parlaments implizieren Effizienzreformen den geringsten Bedarf, kann die Gesetzgebung doch grundsätzlich an die Ministerialbürokratie delegiert werden. Wenn eine parlamentarische Verwaltung aufgebaut wird, bietet es sich an, Handlungsorganisationen auf der Ebene der Fraktionen anzusiedeln. Durch diese Wahl wird sichergestellt, dass praktikable Gesetzesvorschläge ausgearbeitet (bzw. in den Ausschüssen sinnvolle Ergänzungen an Vorschlägen der Regierung angemahnt) werden. Die Ansiedlung auf der Ebene der Fraktionen stellt sicher, dass diese Vorschläge dennoch mit den politischen Vorstellungen der jeweiligen Parteien vereinbar sind. Anders als Legitimitäts- und Effektivitätsreformen sind Maßgaben der Effizienz zudem mit einer engen Kopplung der parlamentarischen Arena an ihren institutionellen Kontext kompatibel. Diese enge Kopplung stellt sicher, dass Gesetzgebungsvorhaben im Fall von Politikverflechtung in Mehrebenensystemen zügig umgesetzt werden können. Sollten überhaupt signifikante Rechte parlamentarischer Minderheitsakteure vorgesehen sein, so sinkt damit die Wahrscheinlichkeit, dass diese angewendet werden. 


\section{Die Sequenzierung von Legitimitäts-, Effektivitäts- und Effizienzreformen als Determinante der parlamentarischen Agendamacht}

Wenn parlamentarische Agendamacht unter Maßgaben der Legitimität, Effektivität und Effizienz verteilt werden kann (Abschnitt 3) und diese Maßgaben eine unterschiedliche Verteilung der Agendamacht implizieren (Abschnitt 4), dann lässt sich folgern, dass das Zusammenspiel der drei Reformarten über die Ausgestaltung der Agendamacht entscheidet. Dieses Zusammenspiel von Legitimitäts-, Effektivitäts- und Effizienzreformen lässt sich genauer als deren zeitliche Abfolge, also die Sequenzierung der jeweiligen Reformtypen, beschreiben. Um die Ausgestaltung der Agendamacht zu untersuchen, gilt es also, die Sequenzierung von Legitimitäts- Effektivitäts- und Effizienzreformen zu ermitteln. Die zeitliche Abfolge von $\mathrm{x}_{1}$ (Legitimitätsreformen), $\mathrm{x}_{2}$ (Effektivitätsreformen) und $\mathrm{x}_{3}$ (Effizienzreformen) ergibt nach dieser Lesart y (die Agendamacht). In empirischer Hinsicht gilt es also die Dynamik der Sequenzierung dieser drei Reformarten zu ermitteln. Werden Legitimitäts-, Effektivitäts- und Effizienzreformen gleichzeitig oder zeitlich klar voneinander getrennt vorgenommen? Die wichtige Rolle der Sequenzierung erklärt, warum die Ausgestaltung der parlamentarischen Agendamacht nur im Zeitverlauf untersucht werden kann, denn nur so lassen sich selbstverstärkende oder reaktive Prozesse ermitteln (Mahoney 2000). Für Analysen der parlamentarischen Agendamacht bedeutet dies, der Frage nachzugehen, ob Legitimitätsund Effektivitäts- bzw. Effizienzreformen im Wechsel aufeinander folgen (wie im Fall reaktiver Prozesse). Alternativ können Präferenzen, die sich historisch herausgebildet haben, einfach fortgeschrieben werden (wie im Fall selbstverstärkender Prozesse). Die Dynamik von Legitimitäts-, Effektivitäts- und Effizienzreformen bestimmt über den kausalen Mechanismus, die der Ausgestaltung der Agendamacht zugrunde liegt (s. a. Grzymala-Busse 2011).

Faktisch dürfte es für die Ausgestaltung der Agendamacht einen großen Unterschied darstellen, ob allein unter einzelnen Gesichtspunkten entschieden wird, ob also Legitimitäts-, Effektivitäts- und Effizienzreformen sequenziell vorgenommen werden, oder ob die Ausgestaltung der Agendamacht gleichzeitig mehreren Leitmotiven folgt. Schaubild 3 verdeutlicht, dass die Konsequenzen der einzelnen Reformarten vor allem zwischen Legitimitäts- und Effizienzreformen deutlich variieren. Wenn also Reformen beispielsweise nicht vorgenommen werden, um allein die Effizienz des Parlaments zu gewährleisten, sondern auch dessen Legitimität, dann dürfte dies die in Schaubild 3 dargestellten Folgen von Effizienzreformen gleichsam abschwächen: $\mathrm{Zu}$ erwarten wären dann erstens eine proportionalere Verteilung der Plenarzeit als bei ausschließlichen Effizienzreformen, zweitens die Ansiedlung von Handlungsorganisationen auf der überparteilichen Ebene (z. B. anstelle der Fraktionen bei den in diesem Fall generell wichtigeren Ausschüssen) und drittens eine losere Kopplung der parlamentarischen Arena an ihren institutionellen Kontext, die es erlauben würde, Rechte parlamentarischer Minderheitsakteure in höherem Maße faktisch anzuwenden.

Die hier zugrundeliegende Annahme, dass einander potenziell überlappende Konflikte die Ausgestaltung der Agendamacht erklären können, lässt auf zyklische Entwicklungen schließen. Aufgrund der in der Regel multiplen Interessen parla- 
mentarischer Akteure, die sich aus deren Heterogenität ergeben (Schickler 2001, S. 15-16), ist nicht zu erwarten, dass eines der drei Leitmotive, denen die Verteilung der Agendamacht folgt, im Zeitverlauf verschwindet. Legitimitäts-, Effektivitäts- und Effizienzprobleme dürften sich vielmehr immer wieder neu stellen. Die Wahrscheinlichkeit von zyklischen Entwicklungen stellt ein weiteres Argument für Langzeitanalysen dar, die wiederum die Aufmerksamkeit auf das Timing von Reformen der parlamentarischen Agendamacht lenken. Das Timing wird als die Platzierung bestimmter Reformen in ihrem (exogenen) Kontext verstanden (Grzymala-Busse 2011, S. 1288). Die beiden potenziell wichtigsten exogenen Ereignisse für die Entwicklung der Agendamacht dürften zum einen die Parlamentarisierung und zum anderen die Demokratisierung sein. Der Prozess der Parlamentarisierung, also der Ausweitung der Mitsprache des Parlaments (die nicht notwendigerweise mit demokratischen Reformen einhergehen muss) sorgt grundsätzlich für eine Verknappung parlamentarischer Zeit (vgl. exemplarisch Cox 1987 für Großbritannien). Von Demokratisierungsprozessen hingegen gehen gegenläufige Impulse zur Stärkung der parlamentarischen Legitimität aus. Die zeitliche Platzierung von Reformen der Agendamacht im Verhältnis zu diesen beiden Prozessen dürfte also einen maßgeblichen Einfluss auf deren Sequenzierung haben. Um die Dynamik zwischen dem Timing und der Sequenzierung von Legitimitäts-, Effektivitätsund Effizienzreformen analysieren zu können, stellt die nach 1870 einsetzende Ausweitung des politischen Wettbewerbs (Caramani 2004, S. 81) einen geeigneten Ausgangspunkt für Langzeitanalysen parlamentarischer Agendamacht dar.

Wenn die jüngere Literatur zu Parlamentsreformen (Sieberer et al. 2011; Blumenthal 2009; Flinders 2007; Kelso 2009; Marschall 1999a) sich also allein auf die Phase nach 1945 bezieht, so gerät damit eine wichtige unabhängige Variable (nämlich die Sequenzierung von früheren Entscheidungen über die parlamentarische Agendamacht) aus dem Blick. Ähnlich wie Patzelt (2012) möchte ich dafür plädieren, die Evolution von Parlamenten zu untersuchen - allerdings systematisch im Hinblick auf die Ausgestaltung der Agendamacht. Das Konzept der Agendamacht eignet sich sehr gut zu diesem Zweck, eben weil das Problem der Verteilung parlamentarischer Zeit spätestens dann virulent ist, wenn Prozesse der Parlamentarisierung und/oder Demokratisierung einsetzen. Unabhängig vom Wandel des jeweiligen Kontextes liegen hier also vergleichbare empirische Ereignisse (Entscheidungen über die Ausgestaltung der Agendamacht) vor, die eine politikwissenschaftliche Langzeitanalyse ermöglichen.

\section{Parlamentarische Agendamacht und normative Befunde der Parlamentarismusforschung}

Nachdem nun die empirischen Möglichkeiten erörtert wurden, die sich aus dem Konzept der Agendamacht ergeben, soll im Folgenden der Nutzen für die normative Debatte über die Zukunft des Parlamentarismus thematisiert werden. Im Mittelpunkt dieses Abschnitts steht die Frage, welchem Primat Reformen der parlamentarischen Agendamacht nach der Lesart dreier normativer Szenarien folgen. Welche Rolle spielen Legitimität, Effektivität und Effizienz für die Befunde einer 
Entparlamentarisierung, des Übergangs in den Postparlamentarismus und in den Neoparlamentarismus?

Der Befund einer Entparlamentarisierung weist darauf hin, dass Parlamente ihre Existenz nur um den Preis von Einbußen bei der Legitimität, Effektivität und der Effizienz gewährleisten können. Parlamente bleiben zwar die Orte, an denen Gesetze verabschiedet werden, allerdings sind die Möglichkeiten, deren Inhalte zu beeinflussen, ebenso begrenzt wie die zur Kontrolle der Regierung oder des Einwirkens auf die öffentliche Meinung. Stattdessen ist der Gesetzgebungsprozess ausgelagert und wird maßgeblich von der Ministerialbürokratie, ad hoc eingesetzten Kommissionen und Verbänden beeinflusst (Schüttemeyer 2007). Darunter leiden nach dieser Lesart sowohl das Ansehen von Parlamenten (d. h. ihre Legitimität) als auch ihre Fähigkeit, die Verantwortlichkeit der Regierung einzufordern (d. h. ihre Effektivität). Vor diesem Hintergrund erscheinen auch Effizienzreformen mit dem Ziel, die Mitsprache des Parlaments bei der Gesetzgebung zu erhöhen, faktisch aussichtlos. Der Begriff der Entparlamentarisierung impliziert also, dass Parlamente insgesamt an Bedeutung verlieren (Schaubild 4). Ähnlich wie die Verteilung parlamentarischer Zeit und die organisatorischen Ressourcen bleiben auch die formalen Regeln im Lichte des Befunds einer Entparlamentarisierung unverändert. Der Unterschied ist, dass die Regeln (und damit Parlamente an sich) faktisch keine Rolle mehr spielen. Eine Entparlamentarisierung wäre damit ein Beispiel institutioneller Konversion, in der die alten Regeln zwar in Kraft bleiben, allerdings anders angewendet werden (Mahoney u. Thelen 2010, S. 17-18).

\section{Schaubild 4: Normative Befunde über Parlamente und die Verteilung von Agendamacht}

\begin{tabular}{|c|c|c|c|}
\hline & $\begin{array}{l}\text { Ent- } \\
\text { parlamentarisierung }\end{array}$ & $\begin{array}{c}\text { Post- } \\
\text { parlamentarismus }\end{array}$ & $\begin{array}{c}\text { Neo- } \\
\text { parlamentarismus }\end{array}$ \\
\hline $\begin{array}{l}\text { Bedeutung der } \\
\text { Leitmotive für die } \\
\text { Ausgestaltung der } \\
\text { Agendamacht* }\end{array}$ & $\begin{array}{c}\text { Legitimität }(-) \\
\text { Effektivität }(-) \\
\text { Effizienz }(-)\end{array}$ & $\begin{array}{c}\text { Legitimität }(+) \\
\text { Effektivität }(-) \\
\text { Effizienz }(-)\end{array}$ & $\begin{array}{c}\text { Legitimität }(+) \\
\text { Effektivität }(+) \\
\text { Effizienz }(-)\end{array}$ \\
\hline $\begin{array}{l}\text { Verteilung parla- } \\
\text { mentarischer Zeit }\end{array}$ & unverändert & $\begin{array}{l}\text { Fokus auf allgemei- } \\
\text { ne Aussprachen: } \\
\text { proportionaler }\end{array}$ & $\begin{array}{l}\text { Fokus auf allgemei- } \\
\text { ne Aussprachen und } \\
\text { Kontrolle der Regie- } \\
\text { rung: proportionaler }\end{array}$ \\
\hline $\begin{array}{l}\text { Organisatorische } \\
\text { Ressourcen des } \\
\text { Parlaments }\end{array}$ & unverändert & $\begin{array}{l}\text { Handlungs- } \\
\text { organisation }\end{array}$ & $\begin{array}{c}\text { Handlungs- und } \\
\text { politische } \\
\text { Organisation }\end{array}$ \\
\hline $\begin{array}{l}\text { Anwendung der } \\
\text { Regeln }\end{array}$ & $\begin{array}{l}\text { Regeln spielen ange- } \\
\text { sichts des Bedeu- } \\
\text { tungsverlusts des } \\
\text { Parlaments keine } \\
\text { Rolle mehr }\end{array}$ & $\begin{array}{l}\text { Rechte parlamen- } \\
\text { tarischer Akteure } \\
\text { werden tendenziell } \\
\text { aufgewertet }\end{array}$ & $\begin{array}{l}\text { Rechte parlamen- } \\
\text { tarischer Akteure } \\
\text { werden tendenziell } \\
\quad \text { aufgewertet }\end{array}$ \\
\hline $\begin{array}{l}\text { Art institutionellen } \\
\text { Wandels }\end{array}$ & $\begin{array}{l}\text { Umwandlung } \\
\text { (conversion) }\end{array}$ & $\begin{array}{c}\text { Ersetzung } \\
\text { (displacement) }\end{array}$ & $\begin{array}{l}\text { Schichtung } \\
\text { (layering) }\end{array}$ \\
\hline
\end{tabular}

* (-) bedeutet, dass ein Reformziel an Wichtigkeit verliert, (+) hingegen, dass es wichtiger wird. 
Auch die Thesen vom Post- und Neoparlamentarismus stimmen darin überein, dass die Effizienz von Parlamenten kaum noch als Reformmotiv taugt. Hier wird mit der zusehends engeren Kopplung zwischen nationalen Parlamenten und supra- bzw. internationalen Entscheidungsarenen argumentiert, aus der ein „exekutiver Multilateralismus“ (Zürn 2005, S. 138-142) folge, der Regierungen zulasten der sie tragenden parlamentarischen Akteure im Prozess der Gesetzgebung privilegiert. Weil parlamentarische Akteure ihre Regierungen schwerlich daran hindern können, in anderen Entscheidungsarenen Kompromisse einzugehen, bleibt der Einfluss von nationalen Parlamenten auf legislative Aktivitäten begrenzt. Allerdings implizieren die Szenarien eines Post- und eines Neoparlamentarismus eine unterschiedliche Intensität des Wandels.

In der postparlamentarischen Demokratie „erfordert die demokratische Legitimation im kooperativen Staat eine Aufwertung der originären Funktion der Parlamente, nämlich Öffentlichkeit zu schaffen, dies um so mehr, weil ihre Entscheidungs- und Kontrollfunktion beschränkt wird“ (Benz 1998, S. 218). Weil also neben der Effizienz auch die parlamentarische Effektivität nach dieser Lesart kaum gestärkt werden kann, bleibt allein die Option, die Legitimität von Parlamenten zu erhöhen. Aus postparlamentarischer Perspektive besteht die Rolle von Volksvertretungen angesichts des zunehmenden Legitimationsdefizits internationalisierter Politik darin, Diskurse zu organisieren und der Öffentlichkeit gegenüber verantwortlich zu sein (Andersen u. Burns 1996, S. 250). Was bedeutet das für die Ausgestaltung der Agendamacht? Wenn mehr parlamentarische Zeit legitimierenden Diskursen gewidmet werden soll, so erfordert dies zweierlei: erstens eine proportionale Verteilung parlamentarischer Zeit und zweitens den Auf- oder Ausbau von Handlungsorganisationen auf der Ebene des gesamten Parlaments, die den parlamentarischen Akteuren die für legitimierende Diskurse nötigen Informationen bereitstellen. Das postparlamentarische Szenario impliziert also die Ersetzung (displacement, Mahoney u. Thelen 2010, S. 16) bestimmter Regeln (z. B. von Vorrechten der Mehrheitsakteure, die sich aus einem Primat der Effizienz ergaben) durch andere (die es ermöglichen, legitimierende Diskurse zu führen). Indirekte Formen der Einflussnahme von Parlamenten auf Regierungen ermöglichen es, beide lose miteinander zu verkoppeln (Benz 1998, S. 213). Unter dieser Bedingung können neue Regeln dann auch faktisch angewendet werden.

Das neoparlamentarische Szenario impliziert den am wenigsten weitreichenden Wandel von Parlamenten. Die zunehmende Verflechtung des Umfeldes parlamentarischer Akteure stellt nach dieser Lesart kein Problem für deren Fähigkeit zur effektiven Kontrolle von Regierungen dar, sondern eher eine Chance, bietet sie doch die Möglichkeit, auch Parlamente in transnationalen Versammlungen miteinander zu verflechten (Marschall 2005b, S. 334). Darüber hinaus wird die Kontrollfunktion von Parlamenten aus dieser Perspektive nicht zuletzt als Ausgleich für die Verluste im Bereich der Gesetzgebung gestärkt (Marschall 2005a, S. 284288). Parlamentarische Zeit wird also für legitimierende Diskurse und für die Kontrolle der Regierung benötigt. Da ja auch nach dieser Lesart weniger Zeit für Gesetzgebung aufgewendet wird, geht auch mit dem neoparlamentarischen Szenario eine proportionalere Verteilung parlamentarischer Zeit einhergehen. Zudem ist das neoparlamentarische Szenario ebenfalls an eine organisatorische Expan- 
sion geknüpft: Damit die Legitimität und die Effizienz von Parlamenten gestärkt werden können, müssen sowohl Handlungsorganisation (auf der Ebene des Parlaments) als auch politische Organisationen (auf der Ebene der Fraktionen) aufoder ausgebaut werden. Dank der von einer Stärkung der Legitimität und der Effizienz implizierten losen Kopplung zwischen Parlamenten und ihrem institutionellen Kontext ist es möglich, dass die Rechte parlamentarischer Akteure tendenziell aufgewertet werden. Dieser Wandel kann als eine Schichtung (layering, Mahoney u. Thelen 2010, S. 16-17) neuer institutioneller Regeln auf die bereits bestehenden beschrieben werden.

In der Tat kommen jüngere empirische Analysen zu dem Ergebnis, dass wie von den hier vorgestellten normativen Szenarien impliziert nichtlegislative Aktivitäten in westeuropäischen Parlamenten in den letzten Jahrzehnten zugenommen haben (Green-Pedersen 2011). Das hier vorgestellte Konzept der parlamentarischen Agendamacht ermöglicht es, systematisch zu untersuchen, welche Form des Wandels von Parlamenten mit dieser Zunahme nichtlegislativer Aktivitäten einhergehen und ob diese normativ dem Szenario der Entparlamentarisierung, des Post- oder des Neoparlamentarismus entspricht.

\section{Fazit: Auf dem Weg zu einer holistischen Analyse von Parlamenten}

Von Weick (1985, S. 57) stammt der schöne Vorschlag, wissenschaftliche Untersuchungen auf einem Zifferblatt zu verorten: Bei zwölf Uhr ist die Allgemeinheit angesiedelt, bei vier Uhr die Genauigkeit und bei acht Uhr die Einfachheit. Zwischen diesen drei Eigenschaften besteht ein Zielkonflikt. Das Konzept der Agendakontrolle ist dann ein Beispiel für Sechs-Uhr-Forschung: Es ist genau und einfach, aber seine Allgemeinheit ist suspekt. Zumindest wenn es zur Analyse des empirischen und normativen Wandels von Parlamenten (und nicht allein von Gesetzgebungsprozessen) herangezogen werden soll, sind Ergänzungen nötig. In diesem Beitrag habe ich für ,Zehn-Uhr-Forschung' plädiert, also für ein ebenfalls einfaches, aber allgemeineres Konzept (was notwendigerweise auf Kosten von dessen Genauigkeit geht). Analysen der parlamentarischen Agendamacht fragen nach der Verteilung von Plenarzeit und organisatorischen Ressourcen zu deren Nutzung sowie der faktischen Anwendung der Rechte parlamentarischer Akteure. Vor allem berücksichtigt die Perspektive der Agendamacht auch, dass Parlamente indirekten Einfluss auf legislative Aktivitäten nehmen können, indem sie Regierungen kontrollieren und durch legitimierende Diskurse die öffentliche Meinung beeinflussen. Da Zeitprobleme in Parlamenten allgegenwärtig sind (zumindest unter der Bedingung einer Parlamentarisierung und/oder Demokratisierung von politischen Systemen), ist das Konzept der Agendamacht hinreichend holistisch, um sowohl international als auch im Zeitverlauf systematisch vergleichende Analysen von Parlamenten anleiten zu können. Die wichtigste unabhängige Variable zur Erklärung der parlamentarischen Agendamacht ist die Sequenzierung von Reformen zur Stärkung der Legitimität, Effektivität und Effizienz von Parlamenten. Diese Reformen implizieren eine grundsätzlich verschiedene Verteilung von parlamentarischer Zeit und Ressourcen zu deren Nutzung. 
Da in diesem Beitrag aus Platzgründen weitgehend auf eine empirische Anwendung des Konzepts der Agendamacht verzichtet wurde, sollen zum Schluss zumindest Hinweise auf dessen empirischen Nutzen gegeben werden. Erstens könnten die hier formulierten Annahmen der Schlüssel zum Verständnis des (bislang nicht erklärten) empirischen Befunds sein, dass die Agendakontrolle in Parlamenten mit langer demokratischer Tradition majoritärer ist als in jungen Demokratien (Carroll et al. 2006, S. 166). Angesichts einer langen demokratischen Tradition ist es wahrscheinlich, dass verschiedene Reformen zeitlich voneinander unabhängig waren, dass also zunächst Legitimitäts- und dann Effizienzreformen (die eine majoritäre Zeitverteilung ermöglichen) vorgenommen wurden. $\mathrm{Zu}$ diesem Befund passt, dass die Kosten von Parlamenten in älteren Demokratien höher sind als in jüngeren Demokratien (Z'Graggen 2009, S. 100-101), dass also möglicherweise dort, wo sich mehr Zeit bot, die Legitimität von Parlamenten zu festigen, zu diesem Zweck weniger auf organisatorische Ressourcen zurückgegriffen wurde. Zweitens dürfte beispielsweise in den jungen Demokratien Ostmitteleuropas (die ebenfalls ein interessantes Analyseobjekt darstellen) angesichts der Gleichzeitigkeit von Demokratisierung und Parlamentarisierung eine stärker proportionale Verteilung der parlamentarischen Agendamacht anzutreffen sein. Hier sind modulare Reformen (vgl. Beissinger 2007) der Agendamacht zu erwarten, die sich in verschiedenen Kontexten in ähnlichen Konstellationen (hier: Sequenzen) ereignen. Wenn sich der hier angedeutete empirische Zusammenhang zwischen der Sequenzierung von Legitimitäts-, Effektivitäts- sowie Effizienzreformen und der Verteilung parlamentarischer Agendamacht herstellen ließe, wäre dies ein Beleg dafür, dass das Konzept der Agendamacht als Grundlage einer holistischen Analyse von Parlamenten dienen kann.

\section{Literatur}

Andersen, Svein S., und Tom Burns. 1996. The European Union and the Erosion of Parliamentary Democracy: A Study of Post-parliamentary Governance. In The European Union: How Democratic Is It?, Hrsg. Svein S. Andersen und Kjell A. Eliassen, 227-251. London u. a.: Sage.

Auel, Katrin. 2005. Introduction: The Europeanisation of Parliamentary Democracy. Journal of Legislative Studies 11:303-318.

Bartolini, Stefano. 1999. Collusion, Competition and Democracy (Part I). Journal of Theoretical Politics 11:435-470.

Bartolini, Stefano. 2000. Collusion, Competition and Democracy (Part II). Journal of Theoretical Politics 12:33-65.

Beisheim, Marianne, und Achim Brunnengräber. 2008. Das Parlament im Globalisierungsprozess. Zeitschrift für Internationale Beziehungen 15:73-100.

Beissinger, Mark. 2007. Structure and Example in Modular Political Phenomena. Perspectives on Politics 5:259-276.

Benz, Arthur. 1998. Postparlamentarische Demokratie? Demokratische Legitimation im kooperativen Staat. In Demokratie - eine Kultur des Westens? 20. Wissenschaftlicher Kongress der Deutschen Vereinigung für Politische Wissenschaft, Hrsg. Michael Greven, 201-222. Opladen: Westdeutscher Verlag. 
Blumenthal, Julia von. 2009. Schach dem Premier! Parlamentsreformen in parlamentarischen Regierungssystemen. In Müssen Parlamentsreformen scheitern? Hrsg. Julia von Blumenthal und Stephan Bröchler, 11-46. Wiesbaden: VS Verlag für Sozialwissenschaften.

Borchert, Jens. 2003. Professional Politicians: Towards a Comparative Perspective. In The Political Class in Advanced Democracies, Hrsg. Jens Borchert und Jürgen Zeiss, 1-25. Oxford: Oxford University Press.

Brunsson, Nils. 1989. The Organization of Hypocrisy. Talk, Decisions and Actions in Organizations. Chichester: Wiley.

Caramani, Daniele. 2004. The Nationalization of Politics. The Formation of National Electorates and Party Systems in Western Europe. Cambridge: Cambridge University Press.

Carroll, Royce, Gary W. Cox und Mónica Pachón. 2006. How Parties Create Electoral Democracy, Chapter 2. Legislative Studies Quarterly 23:153-174.

Cox, Gary W. 1987. The Efficient Secret. Cambridge: Cambridge University Press.

Cox, Gary W. 2000. On the Effects of Legislative Rules. Legislative Studies Quarterly 25:169-192.

Cox, Gary W. 2006. The Organization of Democratic Legislatures. In The Oxford Handbook of Political Economy, Hrsg. Barry R. Weingast und Donald A. Wittman, 141-161. Oxford: Oxford University Press.

Cox, Gary W., und Matthew D. McCubbins. 2005. Setting the Agenda: Responsible Party Government in the U.S. House of Representatives. Cambridge: Cambridge University Press.

Cox, Gary W., und Matthew D. McCubbins. 2011. Managing Plenary Time: The U.S. Congress in Comparative Perspective. In The Oxford Handbook of the American Congress, Hrsg. Eric Schickler und Frances E. Lee, 451-472. Oxford: Oxford University Press.

Demuth, Christian. 2009. Parlamentarische Reformen als evolutionärer Prozess. Institutionelles Lernen am Beispiel der Reform des Untersuchungsausschussgesetzes des Deutschen Bundestages. In Müssen Parlamentsreformen scheitern? Hrsg. Julia von Blumenthal und Stephan Bröchler, 67-88. Wiesbaden: VS Verlag für Sozialwissenschaften.

Döring, Herbert. 1995. Time as a Scarce Resource: Government Control of the Agenda. In Parliaments and Majority Rule in Western Europe, Hrsg. Herbert Döring, 223-246. Frankfurt a. M./New York: Campus Verlag.

Döring, Herbert. 2005. Worauf gründet sich die Agenda-Setzer-Macht der Regierung? In Mechanismen der Politik: Strategische Interaktion im deutschen Regierungssystem, Hrsg. Steffen Ganghof u. Philip Manow, 109-148. Frankfurt a. M./New York: Campus Verlag.

Flinders, Matthew. 2007. Analysing Reform. The House of Commons, 2001-5. Political Studies 55:174-200.

Flinders, Matthew, und Alexandra Kelso. 2011. Mind the Gap: Political Analysis, Public Expectations and the Parliamentary Decline Thesis. British Journal of Politics and International Relations 13:249-268.

Green-Pedersen, Christoffer. 2010. Bringing Parties Into Parliament: The Development of Parliamentary Activities in Western Europe. Party Politics 16:347-369. 
Grzymala-Busse, Anna. 2011. Time Will Tell? Temporality and the Analysis of Causal Mechanisms and Processes. Comparative Political Studies 44:1267-1297.

Hardin, Russell. 2007. Compliance, Consent and Legitimacy. In The Oxford Handbook of Comparative Politics, Hrsg. Carles Boix und Susan C. Stokes, 236-255. Oxford: Oxford University Press.

Hibbing, John R. 1988. Legislative Institutionalization with Illustrations from the British House of Commons. American Journal of Political Science 32:681-712.

Hoffmann Slagter, Tracy, und Gerhard Loewenberg. 2009. Path Dependence as an Explanation of the Institutional Stability of the German Parliament. German Politics 18:469484.

Honneth, Axel. 2011. Verwilderungen. Kampf um Anerkennung im frühen 21. Jahrhundert. Aus Politik und Zeitgeschichte 1-2/2011:37-45.

Huber, John D. 1996. Rationalizing Parliament. Legislative Institutions and Party Politics in France. Cambridge: Cambridge University Press.

Judge, David. 2003. Legislative Institutionalization: A Bent Analytical Arrow? Government and Opposition 38:497-516.

Katz, Richard S., und Peter Mair. 1993. The Evolution of Party Organizations in Europe. The Three Faces of Party Organization. American Review of Politics 14:593-617.

Kelso, Alexandra. 2009. Parliamentary Reform at Westminster. Manchester: Manchester University Press.

Kreppel, Amie. 2011. Legislatures. In Comparative Politics, Hrsg. Daniele Caramani, 159187. Oxford: Oxford University Press.

Laver, Michael, und Kenneth A. Shepsle. 1996. Making and Breaking Governments. Cabinets and Legislatures in Parliamentary Democracies. Cambridge: Cambridge University Press.

Mahoney, James. 2000. Path Dependence in Historical Sociology. Theory and Society 29:507-548.

Mahoney, James, und Kathleen Thelen. 2010. A Theory of Gradual Institutional Change. In Explaining Institutional Change. Ambiguity, Agency, and Power, Hrsg. dies., 1-37. Cambridge: Cambridge University Press.

Majone, Giandomenico. 2006. Agenda Setting. In The Oxford Handbook of Public Policy, Hrsg. Michael Moran, Martin Rein und Robert E. Goodin, 228-250. Oxford: Oxford University Press.

March, James G., und Johan P. Olsen. 1995. Democratic Governance. New York: Free Press.

Marschall, Stefan. 1999a. Parlamentsreform. Ziele, Akteure, Prozesse. Opladen: Leske + Budrich.

Marschall, Stefan. 1999b. Öffentlichkeit und Volksvertretung: Theorie und Praxis der Public Relations von Parlamenten. Opladen: Leske + Budrich.

Marschall, Stefan. 2005a. Parlamentarismus. Eine Einführung. Baden-Baden: Nomos.

Marschall, Stefan. 2005b. Transnationale Repräsentation in Parlamentarischen Versammlungen. Demokratie und Parlamentarismus jenseits des Nationalstaats. BadenBaden: Nomos.

Mattson, Ingvar, und Kaare Strøm. 1995. Parliamentary Committees. In Parliaments and Majority Rule in Western Europe, Hrsg. Herbert Döring, 249-307. Frankfurt a. M./ New York: Campus Verlag. 
Meyer, John W., und Brian Rowan. 1977. Institutionalized Organizations. Formal Structure as Myth and Ceremony. American Journal of Sociology 83:340-363.

McCubbins, Matthew D. 2005. Legislative Process and the Mirroring Principle. In Handbook of New Institutional Economics, Hrsg. Claude Ménard und Mary M. Shirley, 123-148. Dordrecht: Springer.

McGann, Anthony J. 2006. Social Choice and Comparing Legislatures. Constitutional versus Institutional Constraints. Journal of Legislative Studies 12:443-461.

Müller, Wolfgang C. 2002. Parties and the Institutional Framework. In Political Parties in the New Europe, Hrsg. Kurt Richard Luther und Ferdinand Müller-Rommel, 249-292. Oxford: Oxford University Press.

Patzelt, Werner J. 2001. Parlamente und ihre Symbolik. Aufriss eines Forschungsfeldes. In Parlamente und ibre Symbolik. Programm und Beispiele institutioneller Analyse, Hrsg. ders., 39-76. Wiesbaden: Westdeutscher Verlag.

Patzelt, Werner J. 2005. Phänomenologie, Konstruktion und Dekonstruktion von Parlamentsmacht. In Parlamente und ibre Macht. Kategorien und Fallbeispiele institutioneller Analyse, Hrsg. ders., 255-302. Baden-Baden: Nomos.

Patzelt, Werner J. (Hrsg.). 2012. Parlamente und ibre Evolution - Forschungskontext und Fallstudien. Baden-Baden: Nomos (im Erscheinen).

Polsby, Nelson W. 1968. The Institutionalization of the House of Representatives. American Political Science Review, 62:144-168.

Powell, G. Bingham. 2000. Elections as Instruments of Democracy: Majoritarian and Proportional Visions. New Haven: Yale University Press.

Risse, Thomas. 2000: „Let's argue!“ Communicative Action in World Politics. International Organization 54:1-39.

Russo, Federico, und Matti Wiberg. 2010. Parliamentary Questioning in 17 European Parliaments: Some Steps towards Comparison. Journal of Legislative Studies 16:215-232.

Scharpf, Fritz W. 2000. Interdependence and Democratic Legitimation. In Disaffected Democracies. What's Troubling the Trilateral Countries?, Hrsg. Susan J. Pharr und Robert D. Putnam, 101-120. Princeton: Princeton University Press.

Schickler, Eric. 2001. Disjointed Pluralism. Institutional Innovation and the Development of the U.S. Congress. Princeton: Princeton University Press.

Schmidt, Vivien A., und Claudio Radaelli. 2004. Policy Change and Discourse in Europe: Conceptual and Methodological Issues. West European Politics 27:183-210.

Schnapp, Kai-Uwe. 2004. Ministerialbürokratien in westlichen Demokratien. Eine vergleichende Analyse. Opladen: Leske + Budrich.

Schnapp, Kai-Uwe, und Philipp Harfst. 2005. Parlamentarische Informations- und Kontrollressourcen in 22 westlichen Demokratien. Zeitschrift für Parlamentsfragen 36:348-370.

Schüttemeyer, Suzanne S. 2007. Modewort oder Alarmsignal? Befunde und Überlegungen zur Entparlamentarisierung. In Res publica semper reformanda. Wissenschaft und politische Bildung im Dienste des Gemeinwohls, Hrsg. Werner J. Patzelt, Martin Sebaldt und Uwe Kranenpohl, 240-253. Wiesbaden: VS Verlag für Sozialwissenschaften.

Schwarz, Daniel, André Bächtiger und Georg Lutz. 2011. Switzerland: Agenda Setting Power of Government in a Separation-of-Powers Framework. In The Role of Governments in Legislative Agenda Setting, Hrsg. Bjørn Erik Rasch und George Tsebelis, 127 143. London/New York: Routledge. 
Sheingate, Adam. 2010. Rethinking Rules: Creativity and Constraint in the US House of Representatives. In Explaining Institutional Change. Ambiguity, Agency, and Power, Hrsg. James Mahoney und Kathleen Thelen, 168-203. Cambridge: Cambridge University Press.

Sieberer, Ulrich. 2006. Agenda Setting in the German Bundestag. A Weak Government in a Consensus Democracy. German Politics 15:49-72.

Sieberer, Ulrich. 2010. Parlamente als Wablorgane: parlamentarische Wablbefugnisse und ibre Nutzung in 25 europäischen Demokratien. Baden-Baden: Nomos.

Sieberer, Ulrich. 2011. The Institutional Power of Western European Parliaments: A Multidimensional Analysis. West European Politics 34:731-54.

Sieberer, Ulrich, Wolfgang C. Müller und Maiko Isabelle Heller. 2011. Reforming the Rules of the Parliamentary Game: Measuring and Explaining Changes in Parliamentary Rules in Austria, Germany, and Switzerland, 1945-2010. West European Politics 34:948-975.

Strøm, Kaare. 1990. Minority Government and Majority Rule. Cambridge: Cambridge University Press.

Strøm, Kaare. 1995. Parliamentary Government and Legislative Organisation In Parliaments and Majority Rule in Western Europe, Hrsg. Herbert Döring, 52-82. Frankfurt a. M./New York: Campus Verlag.

Taylor, Andrew J. 2006. Size, Power, and Electoral Systems: Exogenous Determinants of Legislative Procedural Choice. Legislative Studies Quarterly 31:323-345.

Tsebelis, George, und Bjørn Erik Rasch. 2011. Governments and Legislative Agenda Setting. An Introduction. In The Role of Governments in Legislative Agenda Setting, Hrsg. Bjørn Erik Rasch und George Tsebelis, 1-20. London/New York: Routledge.

Weick, Karl E. 1985. Der Prozess des Organisierens. Frankfurt a. M.: Suhrkamp.

Z'Graggen, Heidi. 2009. Die Professionalisierung von Parlamenten im historischen und internationalen Vergleich. Bern u. a.: Haupt.

Zubek, Radoslaw. 2011. Negative Agenda Control and Executive-Legislative Relations in East Central Europe, 1997-2008. Journal of Legislative Studies 17:172-193.

Zürn, Michael. 2005. Global Governance and Legitimacy Problems. In Global Governance and Public Accountability, Hrsg. David Held und Mathias Koenig-Archibugi, 136-163. Oxford: Oxford University Press.

\section{Autorenangaben:}

Dr. Michael Koß,

Universität Potsdam, Wirtschafts- und Sozialwissenschaftliche Fakultät, Professur „Politik und Regieren in Deutschland und Europa“, August-Bebel-Str. 89, 14482 Potsdam, mkoss@uni-potsdam.de 